\title{
Application of TG/DSC Analysis under Variable Gas Phase Composition to Study Order-Disorder Transition in Nonstoichiometric Sr-Dy-Cobaltate
}

\author{
Sergey N. Vereshchagin*a, \\ Vyacheslav A. Dudnikov and Yury S. Orlov ${ }^{\mathrm{b}}$ \\ ${ }^{a}$ Institute of Chemistry and Chemical Technology SB RAS \\ $F R C$ "Krasnoyarsk Scientific Center of the SB RAS" \\ 50/24 Akademgorodok, Krasnoyarsk, 660036, Russia \\ ${ }^{b}$ Kirensky Institute of Physics \\ FRC "Krasnoyarsk Scientific Center of the SB RAS" \\ 50/38 Akademgorodok, Krasnoyarsk, 660036, Russia
}

Received 07.09.2018, received in revised form 22.10.2018, accepted 07.12.2018

\begin{abstract}
For the first time a method of TG/DSC analysis under constant and variable gas phase composition was applied to reveal interconnection between quenching ramp rates and degree of phase transformation of cubic nonstoichiometric disordered perovskite (I) $\operatorname{Sr}(0.8) \mathrm{Dy}(0.2) \mathrm{CoO}(3-d)$ to tetragonal structure (II) with ordered Sr/Dy cations and anion vacancies. It was shown that the transformation is a first order phase transition and the ordering process is kinetically controlled. Based on the experimental transition enthalpy it was found that a formal conversion degree of I to II was 98-30\% under cooling ramp rates 3-99 K/min. Non-linear relationship between the completeness of I-to-II transformation and mobile oxygen characteristics (content and the enthalpy of O-removal) was demonstrated.
\end{abstract}

Keywords: perovskite, order-disorder, phase transition, TG, DSC.

Citation: Vereshchagin S.N., Dudnikov V.A., Orlov Yu.S. Application of TG/DSC analysis under variable gas phase composition to study order-disorder transition in nonstoichiometric Sr-Dy-cobaltate, J. Sib. Fed. Univ. Chem., 2018, 11(4), 507-517. DOI: 10.17516/1998-2836-0095.

(C) Siberian Federal University. All rights reserved

* Corresponding author E-mail address: snv@icct.ru 


\title{
Применение ТГ/ДСК-анализа
}

\section{с программированным составом газовой фазы \\ для изучения фазового перехода порядок-беспорядок \\ в нестехиометрическом Sr-Dy-кобальтате}

\author{
С.Н. Верещагин ${ }^{a}$, В.А. Дудников ${ }^{\tilde{\sigma}}$ Ю.С. Орлов \\ ${ }^{a}$ Институт химии и химической технологии СО РАН \\ ФИЦ «Красноярский научный иентр СО РАН» \\ Россия, 660036, Красноярск, Академгородок, 50/24 \\ ${ }^{6}$ Институт физики им. Л.В. Киренского СО РАН \\ ФИЦ «Красноярский научный иентр СО РАН» \\ Россия, 660036, Красноярск, Академгородок, 50/38
}

\begin{abstract}
Методом термического анализа (ТГ/ДСК) в условиях постоянного и программированного состава газовой фазы изучено влияние скорости закалки на полноту перехода кубического разупорядоченного нестехиометрического перовскита (I) $\operatorname{Sr}(0.8) \operatorname{Dy}(0.2) \mathrm{CoO}(3-d) \quad 6$ тетрагональную модификацию (II) с упорядоченным расположением катионов Sr/Dy и анионных вакансий, а также взаимосвязь степени превращения I в II c количеством мобильного кислорода и его энергетическими характеристиками. Показано, что фазовое превращение протекает как фазовый переход первого рода, проиесс образования структуры II контролируется кинетическими факторами, а использование скоростей охлаждения 3-99 К/мин позволяет получить образиы, формально соответствующие 98-30 \% превращению I в II; при этом как количество подвижного кислорода, так и энтальпия его удаления из образиа нелинейно зависят от полноты перехода $\mathbf{I}$ в II.
\end{abstract}

Ключевые слова: перовскит, порядок-беспорядок, фазовый переход, ТГ, ДСК.

\section{Введение}

Оксиды со структурой перовскита $\left(\mathrm{ABO}_{3}\right)$ являются перспективными системами для создания новых материалов с технически важными свойствами - катализаторов, селективных мембран, электродов топливных элементов, газовых сенсоров [1]. Для получения заданных свойств и их тонкой подстройки широко применяются двойные оксиды $\left(\mathrm{A}_{(1-\mathrm{x})} \mathrm{A}_{\mathrm{x}}{ }^{\prime} \mathrm{B}_{(1-\mathrm{y})} \mathrm{B}_{\mathrm{y}}{ }^{\prime} \mathrm{O}_{6}\right)$, которые предоставляют существенно более широкие возможности для регулирования свойств, реализуемые как за счет варьирования природы катионов А и В, так и путем их различного распределения по соответствующим позициям кристаллической решетки. В последнее время возникает все больше экспериментальных подтверждений того, что наряду с варьированием природы катионов и характера их распределения в решетке существенный выигрыш или даже появление принципиально новых свойств можно достичь путем создания в массиве перовскита доменов различной степени упорядоченности и/или нановключений фаз. Наиболее ярким при- 
мером такого подхода служит создание релаксоров (свинецсодержащих перовскитов), которые имеют чрезвычайно высокие значения диэлектрической константы за счет формирования наноупорядоченных областей в разупорядоченной матрице [2]. Отмечалось также, что в системе $\left(\mathrm{La}_{1 / 3-\mathrm{x}} \mathrm{Li}_{3 \mathrm{x}}\right) \mathrm{NbO}_{3}$ при упорядочении катионов в А-позиции происходило образование многочисленных наноразмерных структур двойникования со значительным объемом межструктурных границ, что благоприятно сказывалось на проводимости материала [3]. Тонкое диспергирование на поверхности $(\mathrm{La}, \mathrm{Sr}) \mathrm{CoO}_{3-\delta}$ частиц второй перовскитоподобной фазы $(\mathrm{La}, \mathrm{Sr})_{2} \mathrm{CoO}_{4 \pm \delta}$ приводило к 50-кратному увеличению коэффициента поверхностного кислородного обмена за счет резкого возрастания скорости обмена на границе фаз [4].

Возникновение метастабильных структур локальной неоднородности в твердом теле можно ожидать в области фазовых переходов, когда формирование новой фазы ограничено кинетическими факторами. К настоящему времени накоплен огромный практический материал по физико-химическим свойствам упорядоченных и разупорядоченных перовскитов [5, 6], однако исследования, непосредственно посвященные изучению процесса механизма фазового перехода порядок-беспорядок и обеспечивающие научные основы получения частично разупорядоченных полифазных метастабильных структур, немногочисленны. Одним из наиболее мощных методов получения детальной информации о структуре кристаллов на уровне отдельных атомов и нанодоменов является метод просвечивающей (TEM) и растровой (SEM) электронной микроскопии, а также различные реализации сканирующей туннельной микроскопии (STM, AFM), которые трудоемки и дорогостоящи. Для исследования размера частиц, остаточных напряжений, получения структурной информации также широко применяются методы, основанные на дифракции рентгеновских лучей и нейтронов. Особенности накопления экспериментального сигнала определяют общий недостаток всех вышеперечисленных подходов - затруднения при изучении динамики быстропротекающих процессов, а именно эта информация необходима для целенаправленного поиска условий получения метастабильных систем с неравновесным пространственно неоднородным разупорядочением. В отличие от вышеперечисленных методы термического микроанализа в стандартном исполнении с временем экспериментального отклика около нескольких секунд служат ценным дополнением к комплексу структурных исследований. Поэтому актуальна разработка методик термического анализа для изучения динамики фазовых переходов в твердом теле, для процессов с характеристическими временами от нескольких секунд до десятков минут. Целью настоящей работы являлось изучение возможности применения ТГ/ДСК-анализа в условиях постоянного и программированного состава газовой фазы для изучения динамики процесса фазового перехода порядок-беспорядок на примере нестехиометрического перовскита $\operatorname{Sr}_{0.8} \mathrm{Dy}_{0.2} \mathrm{CoO}_{3-\delta}$.

\section{Экспериментальная часть}

Поликристаллический перовскит $\mathrm{Sr}_{0.8} \mathrm{Dy}_{0.2} \mathrm{CoO}_{3-\delta}$ был получен по стандартной керамической технологии на воздухе при температуре $1473 \mathrm{~K}$ из оксидов $\mathrm{Dy}_{2} \mathrm{O}_{3}, \mathrm{Co}_{3} \mathrm{O}_{4}$ (чистота $99.9 \%$ ) и карбоната стронция $\mathrm{SrCO}_{3}$ (чистота $99 \%$ ), аналогично перовскиту $\mathrm{Sr}_{0.8} \mathrm{Gd}_{0.2} \mathrm{CoO}_{3-\delta}$ [7]. Для получения разупорядоченного кубического $\mathrm{Sr}_{0.8} \mathrm{Dy}_{0.2} \mathrm{CoO}_{3-\delta}$ этот образец подвергался закалке от 1473 К до комнатной температуры (скорость охлаждения в области фазового перехода составляла $\sim 50 \mathrm{~K} / \mathrm{c}$ или 3000 К/мин) с последующей выдержкой 1 ч при 773 К. Синтез упо-

$$
-509-
$$


рядоченного и частично разупорядоченного $\mathrm{Sr}_{0.8} \mathrm{Dy}_{0.2} \mathrm{CoO}_{3-\delta}$ проводился непосредственно в ячейке термического анализатора (ТГ-ДСК). Для этого монолитный фрагмент керамики размером 2 х2х1 мм (масса образца 22.4 мг) нагревался со скоростью 10 К/мин в потоке смеси $20 \% \mathrm{O}_{2}$-Ar до $\mathrm{T}=1473 \mathrm{~K}$, после чего температура линейно снижалась до $\mathrm{T}=773$ К со скоростью $\beta=2-99$ К/мин. Для стабилизации содержания кислорода образец дополнительно выдерживался при этой температуре 1 ч.

Синхронный термический анализ осуществляли на приборе Netzsch STA Jupiter 449C c масс-спектральным анализатором Aeolos QMS 403С в потоке газовой смеси $\mathrm{O}_{2}-\operatorname{Ar}\left(100 \mathrm{~cm}^{3} \mathrm{Mин}^{-1}\right)$, общее давление в ДСК/ТГ ячейке составляло около 1013 кПа. Измерения проводили в платиновых тиглях с перфорированной крышкой. Коэффициент чувствительности сенсора определялся из теплоемкости стандартного сапфирового диска по методу [8], ошибка определения $\Delta \mathrm{H}$ составляла $4 \%$.

Содержание кислорода в исходных перовскитах $\mathrm{Sr}_{0.8} \mathrm{Dy}_{0.2} \mathrm{CoO}_{3-\delta}$ определено из убыли массы образца при его восстановлении водородом по методике [9], погрешность определения нестехиометрии $\delta$ равна \pm 0.01 . Изменение $\delta$ в экспериментах со сменой составов смесей рассчитано на основании изменения массы образца.

Определение количества подвижного кислорода и удельной энтальпии удаления $\mathrm{O}_{2}$ в зависимости от степени упорядоченности $\mathrm{Sr}_{0.8} \mathrm{Dy}_{0.2} \mathrm{CoO}_{3-\delta}$ проведено «импульсным» методом с фиксацией вариации массы и теплового потока при ступенчатом изменении состава продувочных газов в ячейке прибора термического анализа в последовательности (\% об.): $13 \% \mathrm{O}_{2}-\mathrm{Ar}=>100 \% \mathrm{Ar}=>13 \% \mathrm{O}_{2}$-Ar; детали эксперимента подробно описаны в работе [10].

Исходные данные для рентгеноструктурного анализа получены с использованием дифрактометра PANalytical X'Pert PRO (CoK $\alpha)$ в интервале углов $2 \theta 10-140^{\circ}$. Обработка результатов проведена аналогично исследованию перовскита $\mathrm{Sr}_{0.8} \mathrm{Gd}_{0.2} \mathrm{CoO}_{3-\delta}$ [11].

\section{Результаты и обсуждение}

Получение частично разупорядоченного по А-позищиям перовскита $\mathrm{Sr}_{0.8} \mathrm{D} \mathrm{y}_{0.2} \mathrm{CoO}_{3-\delta}$ и его исследование методом термического анализа

Поведение $\mathrm{Sr}_{0.8} \mathrm{Dy}_{0.2} \mathrm{CoO}_{3-\delta}$ перовскита в циклах нагревания-охлаждения было аналогично поведению Sr-Gd-системы, изученной нами ранее [11]. После медленного охлаждения (отжига) Sr-Dy-перовскита от 1473 К со скоростью 2 K/мин в смеси $20 \% \mathrm{O}_{2}$-Аr и выдерживании 1 ч при $773 \mathrm{~K}$ для стабилизации содержания кислорода его состав соответствовал формуле $\mathrm{Sr}_{0.8} \mathrm{Dy}_{0.2} \mathrm{CoO}_{2.63}$ $(\delta=0.37)$. По данным рентгеноструктурного анализа, отожженный $\mathrm{Sr}_{0.8} \mathrm{Dy}_{0.2} \mathrm{CoO}_{2.63}$ представлял собой монофазную керамику с тетрагональной $I 4 / \mathrm{mmm}$ суперструктурой II с упорядоченным расположением А-катионов, аналогичной описанной в работе [12]. В ней присутствовали три неэквивалентные позиции А-катионов, в одной из которых (A1) статистически распределены ионы $\mathrm{Sr}^{2+} / \mathrm{Dy}^{3+}$, а две оставшиеся (A2, А3) занимали исключительно ионы $\mathrm{Sr}^{2+}$. Анионные вакансии в данной структуре при 298 К располагались только в одной из четырех неэквивалентных позиций (рис. 1). При быстром охлаждении (закалке, 50 К/с) от 1473 до 298 К сохранялась высокотемпературная модификация, которая при комнатной температуре представляла собой монофазный разупорядоченный нестехиометрический кубический Pm3m перовскит I состава 


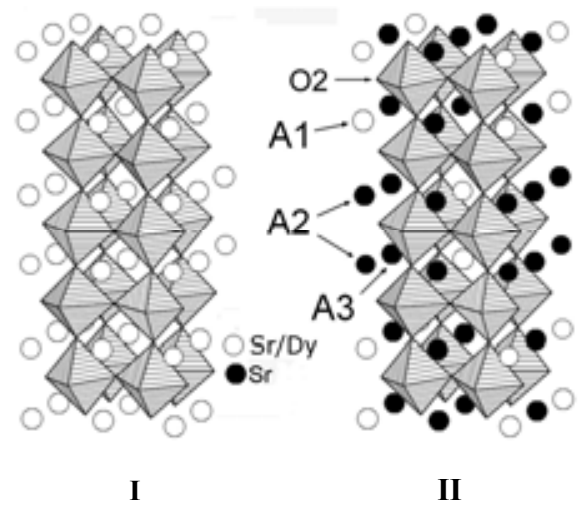

Рис. 1. Изображение разупорядоченной кубической (I) и упорядоченной тетрагональной (II) структуры $\mathrm{Sr}_{0.8} \mathrm{Dy}_{0.2} \mathrm{CoO}_{3-\delta}$. Фрагментам $\mathrm{CoO}_{6}$ соответствуют октаэдры, в вершинах которых расположены ионы $\mathrm{O}^{2-}$ и анионные вакансии. Белые сферы обозначают позиции $\mathrm{Sr}^{2+}$, черные - позиции катионов $\mathrm{Sr}^{2+} / \mathrm{Dy}^{3+}$, метками A1-A3 обозначены неэквивалентные позиции А-катионов, O2 - позиция преимущественной локализации кислородных вакансий

Fig. 1. The structure of ordered cubic (I) and disordered tetragonal (II) $\operatorname{Sr}_{0.8} \mathrm{Dy}_{0.2} \mathrm{CoO}_{3-\delta}$. Octahedra represent $\mathrm{CoO}_{6}$, white spheres represent $\mathrm{Sr}^{2+}$, black spheres represent $\mathrm{Sr}^{2+} / \mathrm{Dy}^{3+}$. A1-A3 mark A-cation positions, and $\mathrm{O} 2$ marks positions of anion vacancies

$\mathrm{Sr}_{0.8} \mathrm{Dy}_{0.2} \mathrm{CoO}_{2.73}(\delta=0.27)$ со случайным распределением катионов $\mathrm{Sr}^{2+} / \mathrm{Dy}^{3+}$ и анионных вакансий по соответствующим позициям кристаллической решетки (рис. 1).

Согласно данным термического анализа, заметная подвижность кислорода в структуpe упорядоченного $\mathrm{Sr}_{0.8} \mathrm{Dy}_{0.2} \mathrm{CoO}_{2.63}$, вызывающая изменение массы образца при увеличении/ снижении температуры (рис. $2 a$ ), наблюдалась при Т $>773 \mathrm{~K}$ и происходила в соответствии с уравнением (1). Эндотермический пик на кривой ДСК в интервале 1280-1404 К (температура максимума $\left.\mathrm{T}_{\mathrm{D}}=1400 \mathrm{~K}, 20 \% \mathrm{O}_{2}, \beta=10 \mathrm{~K} / \mathrm{Mин}\right)$ соответствовал переходу упорядоченной структуры II в разупорядоченную модификацию I (od-переход). Обратный процесс упорядочения (do-переход) сопровождался выделением тепла и наблюдался при 1338-1247 ( $\mathrm{T}_{\mathrm{O}}=1309 \mathrm{~K}, 20$ \% $\mathrm{O}_{2}, \beta=-10 \mathrm{~K} /$ мин, рис. $2 a$ ). Эти фазовые превращения протекали на фоне постоянного плавного изменения стехиометрии за счет выделения кислорода, коэффициент нестехиометрии $\delta$ при температуре od- и do-перехода составил 0.46 и 0.44 соответственно.

$$
\mathrm{Sr}_{0.8} \mathrm{Dy}_{0.2} \mathrm{CoO}_{3-\delta 1} \leftrightarrow \mathrm{Sr}_{0.8} \mathrm{Dy}_{0.2} \mathrm{CoO}_{3-\delta 2}+\left(\delta_{2}-\delta_{1}\right) / 2 \mathrm{O}_{2}+\Delta \mathrm{H}
$$

Изменение скорости сканирования $\beta$ по-разному сказывалось на температурах od- и doпереходов. Температура максимума пика $\mathrm{T}_{\mathrm{D}}$ для процесса $\mathbf{I I} \rightarrow \mathbf{I}$ оставалась практически постоянной при росте $\beta$, тогда как величина $\mathrm{T}_{\mathrm{O}}(\mathbf{I} \rightarrow \mathbf{I I})$ снижалась при увеличении скорости охлаждения (рис. 2b). Как следует из литературы, зависимость положения ДСК пика от скорости изменения температуры $\beta$ может как быть следствием методических особенностей проведения анализа, связанных с наличием термосопротивления в системе образец-тигель-сенсор, так и отражать особенности кинетики процессов образования и роста зародышей [13]. Для учета влияния процессов переноса тепла на положение пика была предложена методика [13], основанная на анализе зависимости температуры максимума ДСК-пика $T_{m}$ от $\beta^{1 / 2}$. Экстраполяция функции $\mathrm{T}_{\mathrm{m}}=f\left(\beta^{1 / 2}\right)$ к нулевой скорости сканирования $\beta=0$ позволяет получить «истинные» 

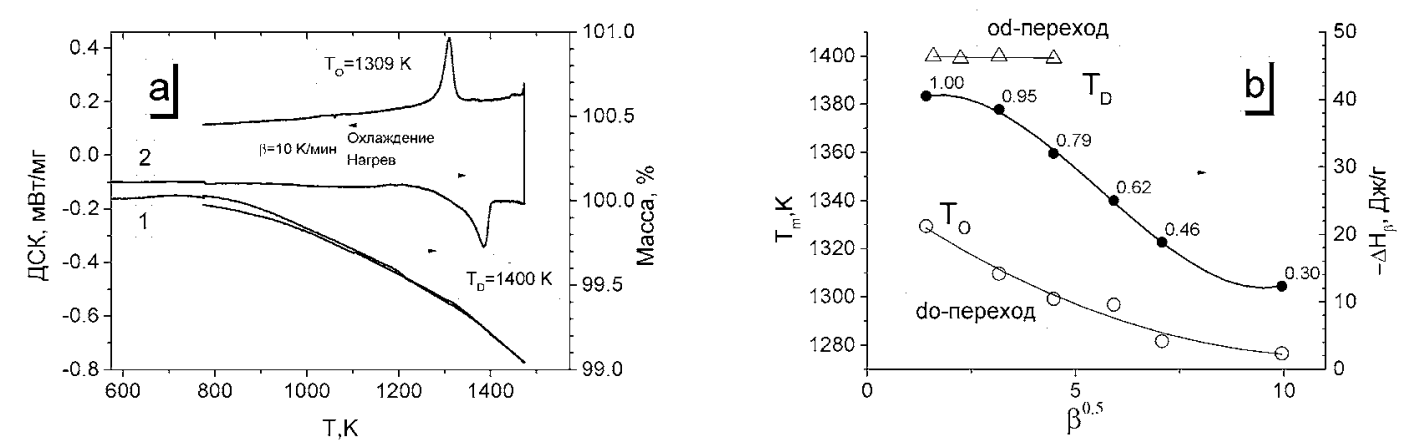

Рис. 2. a - ТГ (1) и ДСК (2) кривые, полученные при нагреве/охлаждении $\mathrm{Sr}_{0.8} \mathrm{Dy}_{0.2} \mathrm{CoO}_{3-\delta}$ в потоке смеси $20 \% \mathrm{O}_{2}-\mathrm{Ar}, \beta=10 \mathrm{~K} /$ мин. $\mathrm{T}_{\mathrm{D}}$ и $\mathrm{T}_{\mathrm{O}}$ - температуры максимума/минимума ДСК-пиков для процессов $\mathbf{I} \rightarrow \mathbf{I I}$ и $\mathbf{I I} \rightarrow \mathbf{I}$ соответственно. b - зависимость температур максимумов пиков $\mathrm{T}_{\mathrm{D}}(\Delta), \mathrm{T}_{\mathrm{O}}(\mathrm{o})$ и энтальпии процесса упорядочения $\Delta \mathrm{H}_{\beta}(\bullet)$ от скорости нагревания $(\Delta)$ и охлаждения $(\bullet, 0)$. Значения около точек $\Delta \mathrm{H}_{\beta}$ соответствуют величине параметра $x$ (полнота превращения $\mathbf{I} \rightarrow \mathbf{I I})$, рассчитанной по (2)

Fig. 2. a - TG (1) and DSC(2) curves for $\mathrm{Sr}_{0.8} \mathrm{Dy}_{0.2} \mathrm{CoO}_{3-\delta}$ heating/cooling cycle $\left(20 \% \mathrm{O}_{2}-\mathrm{Ar}, \beta=10 \mathrm{~K} / \mathrm{min}\right)$. $\mathrm{T}_{\mathrm{D}}$ and $\mathrm{T}_{\mathrm{O}}$ - temperatures of DSC peak extremum for $\mathbf{I} \rightarrow \mathbf{I I}$ and $\mathbf{I I} \rightarrow \mathbf{I}$ phase transitions, respectively. $\mathrm{b}-\mathrm{T}_{\mathrm{D}}(\Delta), \mathrm{T}_{\mathrm{O}}(\mathrm{o})$ and enthalpy of ordering $\Delta \mathrm{H}_{\beta}(\bullet)$ as a function of heating $(\Delta)$ or cooling $(\bullet, 0)$ ramp. Values near $\Delta \mathrm{H}_{\beta}$ points denote a completeness of $\mathbf{I} \rightarrow \mathbf{I I}$ transformation according to equation (2)

значения $\mathrm{T}_{\mathrm{D}}$ и $\mathrm{T}_{\mathrm{O}}$, не искаженные методическими погрешностями. Из приведенных на рис. $2 b$ данных следует, что в точке $\beta=0$ величины $\mathrm{T}_{\mathrm{D}}$ и $\mathrm{T}_{\mathrm{O}}$ существенно различаются, что позволяет уверенно приписать исследуемый процесс к фазовому переходу первого рода, а характер зависимости $\mathrm{T}_{\mathrm{m}}=f\left(\beta^{1 / 2}\right)$, по аналогии co $\mathrm{Sr}-\mathrm{Gd}$-системой [11], указывает на то, что процесс разупорядочения $(\mathbf{I I} \rightarrow \mathbf{I})$ контролируется в значительной степени термодинамическими параметрами, тогда как переход $(\mathbf{I} \rightarrow \mathbf{I I})$ осложнен кинетически. В этом случае логично предположить, что при охлаждении $\mathrm{Sr}_{0.8} \mathrm{Dy}_{0.2} \mathrm{CoO}_{3-\delta}$ от температур выше do-перехода со скоростями в интервале 2-3000 К/мин может формироваться керамика, в которой процесс упорядочения с образованием сформированной фазы II прошел не до конца. Указанием на неполное протекание процесса упорядочения и образование промежуточного «композита» (который может быть как смесью фаз I и II, так и совокупностью доменов, кристаллическая решетка которых разупорядочена в различной степени) может служить величина энтальпии фазового превращения $\Delta \mathrm{H}$, которая в случае фазового перехода первого рода должна зависеть от полноты протекания процесса. Действительно, величина наблюдаемого экзотермического эффекта на кривой ДСК в области 12801330 К снижалась при увеличении скорости охлаждения от значения -40.5 Дж/г при $\beta=-2 К /$ мин

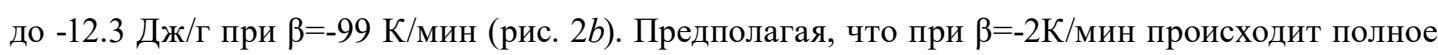
упорядочение, т.е. формируется керамика, состоящая только из упорядоченной фазы II, можно оценить полноту превращения $x$ для образцов, полученных при скорости охлаждения $\beta$ по формуле.

$$
x=\frac{\Delta H_{d o, \beta}}{\Delta H_{d o}}
$$

где $\Delta \mathrm{H}_{\mathrm{do}, \beta}, \Delta \mathrm{H}_{\mathrm{do}}-$ энтальпии процесса при скорости охлаждения $\beta$ и 2 К/мин соответственно.

Рассчитанные таким образом величины $x$ приведены на рис. $2 b$ рядом с точками $\Delta \mathrm{H}_{\mathrm{do}, \beta}$. Из приведенных значений следует, что снижение $\Delta \mathrm{H}$ при использовании различных скоро- 
стей охлаждения в диапазоне 3-99 К/мин формально соответствует формированию системы, в которой полнота протекания процесса $\mathbf{I} \rightarrow \mathbf{I I}$ для исходно разупорядоченного $\mathrm{Sr}_{0.8} \mathrm{Dy}_{0.2} \mathrm{CoO}_{3-\delta}$ составляет 30-95\%.

Влияние частичного разупорядочения $A$-катионов в $\mathrm{Sr}_{0.8} \mathrm{D} \mathrm{y}_{0.2} \mathrm{CoO}_{3-\delta}$

на свойства слабосвязанного кислорода

Для исследования влияния частичного разупорядочения катионов в А-позиции на свойства мобильного кислорода была применена методика термического анализа со ступенчатым изменением состава газовой фазы, разработанная нами ранее [10]. Типичный эксперимент проводился следующим образом: $\mathrm{Sr}_{0.8} \mathrm{Dy}_{0.2} \mathrm{CoO}_{3-\delta}$ нагревался до $1473 \mathrm{~K}(\beta=10 \mathrm{~K} / \mathrm{Mин})$ в потоке смеси $13 \%$ об. О $\mathrm{O}_{2}$-Ar, охлаждался со скоростью $\beta=2-99$ К/мин до $773 \mathrm{~K}$, выдерживался при этой температуре 1 ч, после чего продувочный газ состава $13 \% \mathrm{O}_{2}$-Ar на 1 мин заменялся на чистый аргон с сохранением общего потока, с последующей продувкой исходной смесью $13 \% \mathrm{O}_{2}$-Ar; приведенный цикл повторялся не менее трех раз. Перед экспериментами были проведены холостые циклы с пустыми тиглями, полученные кривые использовались как базовые линии для коррекции изменений массы (ТГ, $\Delta \mathrm{m})$ и теплового потока (ДСК, $\Delta \mathrm{H})$.

Эксперименты показали, что при описанном выше переключении потоков исходно прямоугольный «импульс» аргона размывался при прохождении через ТГ/ДСК систему, профиль концентраций $\mathrm{O}_{2}$ приблизительно соответствовал распределению Гаусса, а его парциальное давление $\left(\mathrm{P}_{\mathrm{O} 2}\right)$ в измерительной ячейке плавно снижалось с 13.2 до 1.3 кПа с последующим увеличением до 13.2 кПа. При этом во время уменьшения $\mathrm{P}_{\mathrm{O} 2}$ (рис. $3 a, b ; \mathrm{t}<\mathrm{t}_{1}$ ) наблюдалось снижение массы образцов за счет реакции (1) с одновременным поглощением теплоты, что отражалось на кривой ДСК в виде эндотермического пика $\left(\Delta \mathrm{H}_{1}\right.$, вставка рис. $\left.3 b\right)$. После прохождения минимума $\mathrm{P}_{\mathrm{O} 2}\left(\mathrm{t}>\mathrm{t}_{1}\right.$, pис. $\left.3 a, b\right)$ начинался процесс реокисления $\mathrm{Sr}_{0.8} \mathrm{Dy}_{0.2} \mathrm{CoO}_{3-\delta 2}$ с поглощением кислорода, выделением теплоты $\Delta \mathrm{H}_{2}$ и восстановлением исходной массы. Следует отметить, что площади эндо- и экзотермических пиков на кривой ДСК, соответствующие потере кислорода и реокислению образца, равны между собой и не изменялись при проведении последовательной серии импульсов, что указывает на обратимость процесса. Полученные таким образом величины $\Delta \mathrm{m}_{\beta}$ (снижение массы в максимуме пика на кривой ТГ, рис. $3 a$ ) и $\Delta \mathrm{H}_{\beta}=\Delta \mathrm{H}_{1} / \Delta \mathrm{m}_{\beta}$ (удельная теплота удаления кислорода) могут быть использованы как характеристики количества подвижного кислорода и прочности его связи с поверхностью соответственно.

Приведенные на рис. 3 ТГ и ДСК кривые однозначно показывают, что скорость охлаждения образцов $\beta$ существенно влияет как на количество мобильного кислорода, так и на энергетику его удаления. Так, изменение массы $\Delta \mathrm{m}$ (рис. $3 a$ ) для образцов $\operatorname{Sr}_{0.8} \mathrm{Dy}_{0.2} \mathrm{CoO}_{3-\delta}$ c разупорядоченной (I, $\beta=3000$ K/мин) и упорядоченной (II, $\beta=2$ K/мин) структурой составляло -0.052 и - 0.019 \% соответственно; в промежутке между этими значениями находились образцы, полученные при скоростях охлаждения $\beta=10-99$ К/мин. Аналогичная картина наблюдалась и для ДСК кривых (рис. 3b).

Представляло интерес проследить, как изменяются наблюдаемые количества активного кислорода и его энергетические характеристики при постепенном преобразовании системы от индивидуальной разупорядоченной фазы I к упорядоченной фазе II. Для количественной характеристики глубины превращения $\mathbf{I} \rightarrow \mathbf{I I}$ будем использовать параметр $x$, рассчитанный по 

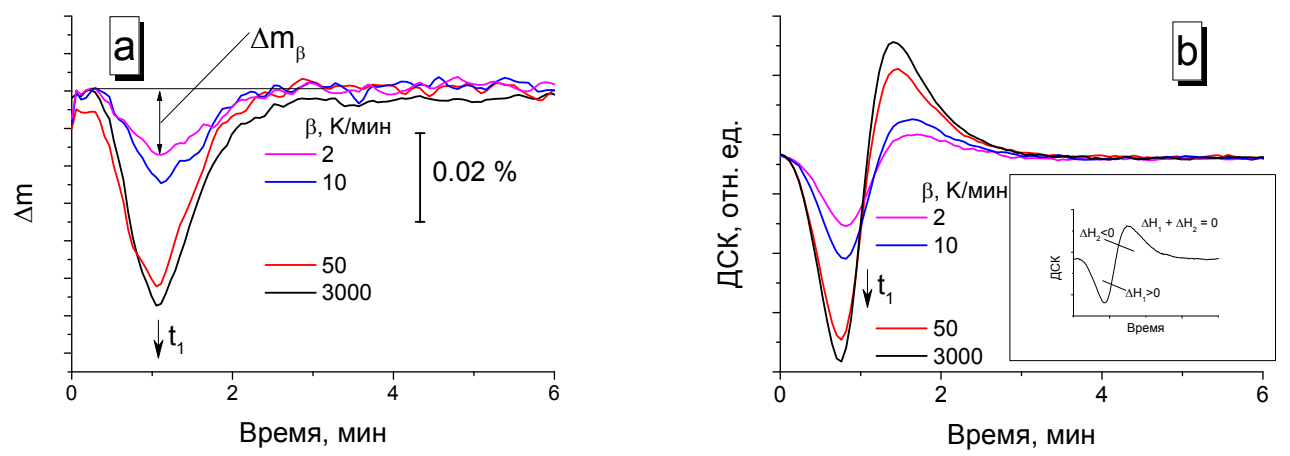

Рис. 3. Вариации массы (а) и ДСК-сигнала (b) при ступенчатом изменении парциального давления кислорода в газовой фазе при $773 \mathrm{~K}$ для серии образцов $\mathrm{Sr}_{0.8} \mathrm{Dy}_{0.2} \mathrm{CoO}_{3-\delta}$, охлажденных от $1473 \mathrm{~K}$ со скоростью 2, 10, 50 и 3000 K/мин. Последовательность подачи смеси: (13\% O $-\mathrm{Ar})-(100 \% \mathrm{Ar}, 1$ мин) (13\% $\mathrm{O}_{2}$-Ar). На вставке проиллюстрирована процедура интегрирования ДСК-пика (см. текст)

Fig. 3. TG (a) and DSC-signal (b) variations under stepwise change of $\mathrm{O}_{2}$ partial pressure at $773 \mathrm{~K}$ for a number of $\mathrm{Sr}_{0.8} \mathrm{Dy}_{0.2} \mathrm{CoO}_{3-\delta}$ samples after cooling from $1473 \mathrm{~K}$ with different ramp rates $(2,10,50$ and $\sim 3000 \mathrm{~K} / \mathrm{min})$. Purging gas: $\left(13 \% \mathrm{O}_{2}\right.$-Ar $)-(100 \% \mathrm{Ar}, 1 \mathrm{~min})-\left(13 \% \mathrm{O}_{2}\right.$-Ar $)$. The inset illustrates DSC curve integration (see text)

уравнению (2), а экспериментальные значения потери массы $\left(\Delta \mathrm{m}_{\beta}\right)$ и удельной энтальпии удаления кислорода $\left(\Delta \mathrm{H}_{\beta}\right)$ будем сравнивать с величинами $\Delta \mathrm{m}_{\text {add }}$ и $\Delta \mathrm{H}_{\text {add }}$, рассчитанными из предположения о линейной зависимости свойств от глубины превращения, или, что то же самое, об аддитивном характере свойств по отношению к свойствам индивидуальных компонентов (уравнения 3a и 3b):

$$
\begin{aligned}
& \Delta m_{\text {add }}=\Delta m_{I}(1-x)+\Delta m_{I I} x, \\
& \Delta H_{\text {add }}=\frac{\Delta m_{I} \Delta H_{I}(1-x)+\Delta m_{I I} \Delta H_{I I} x}{\Delta m_{I}(1-x)+\Delta m_{I I} x},
\end{aligned}
$$

где $x$ - массовая доля упорядоченной фазы II; $\Delta m_{a d d}, \Delta m_{I}, \Delta m_{I I}-$ количество активного кислорода; $\Delta H_{a d d}, \Delta H_{I}, \Delta H_{I I}$ - удельные энтальпии его удаления из смеси (индекс $a d d$ ), разупорядоченной (I) и упорядоченной (II) индивидуальных фаз соответственно.

Сопоставление экспериментальных значений потери массы $\left(\Delta \mathrm{m}_{\beta}\right)$ и удельной энтальпии удаления кислорода $\left(\Delta \mathrm{H}_{\beta}\right)$ с соответствующими расчетными аддитивными величинами $\left(\Delta \mathrm{m}_{\mathrm{add}}\right.$, $\Delta \mathrm{H}_{\text {add }}$ ) показывает, что наблюдается существенное отклонение от аддитивного поведения. Так, для чистых фаз I и II экспериментально определенное количество мобильного кислорода составило $\Delta \mathrm{m}_{\mathrm{I}}=-0.0522 \%, \Delta \mathrm{m}_{\mathrm{II}}=-0.01917 \%$ соответственно, а его превышение над аддитивным значением, рассчитанным по уравнению (3а) для промежуточных образцов со степенью (глубиной) превращения $\mathbf{I} \rightarrow \mathbf{I I} x=0.3-0.8$, находилось в интервале 0.009-0.013 \%, что значительно превышало ошибку определения $\Delta \mathrm{m}$ (рис. 4). Аналогичная, но менее выраженная картина наблюдалась для $\Delta \mathrm{H}_{\mathrm{O} 2}$, причем наиболее заметные отклонения были видны в диапазоне малых содержаний разупорядоченной фазы I.

Как показали теоретические расчеты [14] и экспериментальные исследования [15], подвижность кислорода в перовскитах существенно зависит от характера распределения катионов в А-позиции кристаллической решетки, определяющих и локализацию кислородных вакансий.

$$
-514-
$$



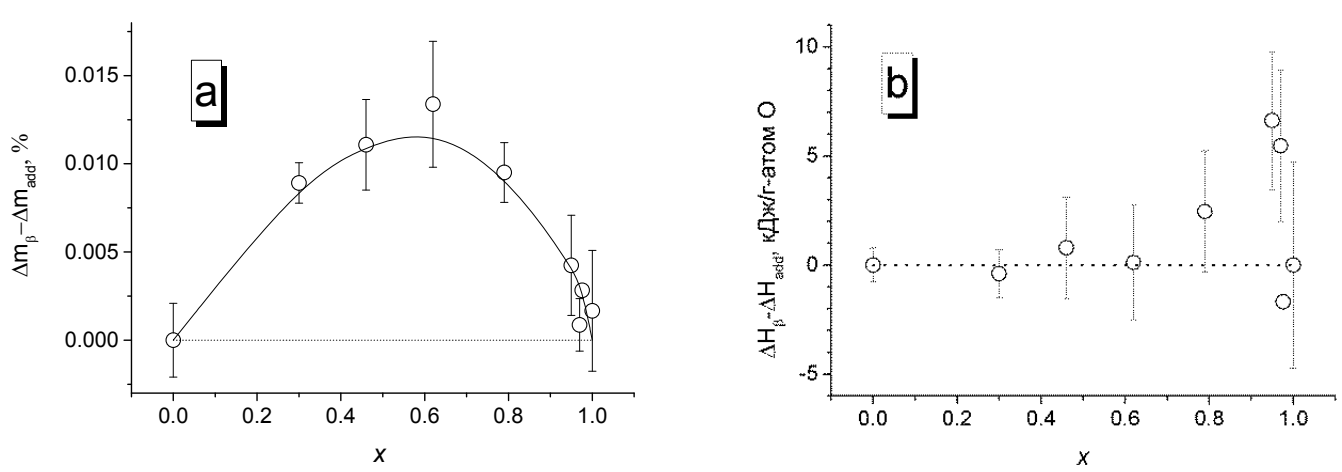

Рис. 4. Отклонение от аддитивного значения содержания подвижного кислорода $\Delta \mathrm{m}_{\beta}$ (a) и удельной энтальпии его удаления $\Delta \mathrm{H}_{\beta}(\mathrm{b})$ при $773 \mathrm{~K}$ в зависимости от параметра $x$ (полноты превращения $\mathbf{I} \rightarrow \mathbf{I I}$ ). Расчет $x, \Delta \mathrm{m}_{\text {mix }}$ и $\Delta \mathrm{H}_{\text {mix }}$ по соотношениям (2),(3a) и (3b) соответственно. Пунктирная линия соответствует аддитивному поведению, отрезки у точек указывают величину стандартного отклонения определения среднего

Fig. 4. Deviations from additive behavior as a function of $x$ (completeness of $\mathbf{I} \rightarrow \mathbf{I I}$ transformation): (a) mobile oxygen amount $\Delta \mathrm{m}_{\beta}$ and (b) specific enthalpy of oxygen removal $\Delta \mathrm{H}_{\beta}$ at $773 \mathrm{~K} . x, \Delta \mathrm{m}_{\text {mix }}$ and $\Delta \mathrm{H}_{\text {mix }}$ are calculated according to (2), (3a) and (3b), respectively. Dotted lines denote additive trends, whiskers are SD-values of mean

Упорядоченное расположение А-катионов сопровождается упорядочением анионных вакансий, что и является причиной различий в подвижности кислорода для индивидуальных фаз, находящихся в упорядоченном или разупорядоченном состоянии. Аналогичный эффект может наблюдаться также в том случае, когда упорядочение кислородных вакансий происходит без изменения А-подрешетки. Так, кислородная проводимость $\mathrm{SrCo}_{0.8} \mathrm{Fe}_{0.2} \mathrm{O}_{3-\delta}$ при температурах вблизи фазового перехода от низкотемпературного состояния с упорядоченными вакансиями к высокотемпературной модификации разупорядоченного кислород-дефицитного перовскита при $\mathrm{T}>1063 \mathrm{~K}$ заметно повышалась, что объяснялось двухфазным характером образца - смеси фаз с упорядоченными и разупорядоченными анионными вакансиями [16].

На основании этого можно было бы предположить, что содержание подвижного кислорода будет пропорционально содержанию разупорядоченной фазы. Однако существенные отклонения от такого аддитивного поведения как по количеству мобильного кислорода, так и по энтальпии его удаления, обнаруженные для исследуемых образцов $\mathrm{Sr}_{0.8} \mathrm{Dy}_{0.2} \mathrm{CoO}_{3-\delta}$ с различной долей упорядочения (рис. 4), свидетельствуют о более сложных отношениях подвижность кислорода - структурное разупорядочение. В частности, такими дополнительными факторами может быть размер доменов с упорядоченным/разупорядоченным распределением катионов/ вакансий, а также наличие и природа межфазных границ, которые, как было показано, могут играть существенную роль в процессах активации и переноса кислорода [4, 17].

\section{Заключение}

Проведенное изучение методом термического анализа ряда перовскитов $\operatorname{Sr}_{0.8} \mathrm{Dy}_{0.2} \mathrm{CoO}_{3-\delta}$ с различной степенью упорядочения катионов $\mathrm{Sr}^{2+} / \mathrm{Dy}^{3+}$ по А-позициям кристаллической решетки (переход порядок-беспорядок) показало, что для подобных систем применение ТГ/ДСК-методик, включающих программируемое изменение состава газовой фазы (парциального давления кислорода), позволяет непосредственно измерять характеристики 
мобильного кислорода в образце, что затруднительно при классическом подходе проведения анализа в среде постоянного состава, а совместное применение классического термического анализа (ТГ/ДСК) с «импульсной» методикой позволило впервые выявить неаддитивное поведение системы $\mathrm{Sr}_{0.8} \mathrm{Dy}_{0.2} \mathrm{CoO}_{3-\delta}$ с частичным превращением исходно полностью разупорядоченного нестехиометрического перовскита в его упорядоченный по А-подрешетке аналог.

\section{Благодарности}

Исследование выполнено при финансовой поддержке Российского фонда фундаментальных исследований, Правительства Красноярского края, Красноярского краевого фонда науки в рамках научного проекта № 18-42-243004 и проекта фундаментальных исследований СО РАН V.45.3.3.

\section{Список литературы}

1. Tao S.W. and Irvine J.T.S. Metal Oxides: Chemistry and Applications, ed. J.L.G. Fierro. Boca Raton: CRC Press, 2006. P. 739.

2. Bhalla A.S., Guo R., Roy R.. The perovskite structure - a review of its role in ceramic science and technology. Mat. Res. Innovat 2000. Vol. 4, P. 3-26.

3. Garcia-Martin S. and Alario-Franco M.A.. Modulated Structure of $\mathrm{La}_{1 / 3-\mathrm{x}} \mathrm{Li}_{3 \mathrm{x}} \mathrm{NbO}_{3} 0 \leq \mathrm{x} \leq 0.06$. J. Solid State Chem 1999. Vol. 148, P. 93-99.

4. Hayd J., Yokokawa Harumi and Ivers-Tiff'ee E.. Hetero-Interfaces at Nanoscaled (La,Sr) CoO3- $\delta$ Thin-Film Cathodes Enhancing Oxygen Surface-Exchange Properties. Journal of The Electrochemical Society 2013. Vol. 160 (4), P. F351-F359.

5. Liu R., Xuan Y., Jia Y.Q. Ordering and disordering in (A'A") (B'B') $\mathrm{O}_{3}$-type perovskite compounds. Materials Chemistry and Physics 1998. Vol. 57, P. 81-85.

6. King G., Woodward P. M.. Cation ordering in perovskites. J. Mater. Chem. 2010. Vol. 20, P. $5785-5796$.

7. Верещагин С.Н., Дудников В.А., Соловьев Л.А. Изучение фазового перехода порядокбеспорядок в нестехиометрическом Sr-Gd-кобальтате методами ДСК, ТГ и РС. Журнал Сuбирского федерального университета. Серия Химия. 2016. Т. 9(3), С. 326-336. [Vereshchagin S., Dudnikov V., Solovyov L.. DSC+TG and XRD study of order-disorder transition in nonstoichiometric Sr-Gd-cobaltate. Journal of Siberian Federal University. Chemistry 2016. Vol. 9(3), P. 326-336. (in Russ.)]

8. Thermal analysis; differential thermal analysis; principles. DIN 51007:1994-06.

9. Conder K., Pomjakushina E., Soldatov A., Mitberg E. Oxygen content determination in perovskite-type cobaltates. Materials research bulletin 2005. Vol. 40, P. 257-263.

10. Верещагин С.Н., Дудников В.А., Соловьев Л.А. Изучение подвижного кислорода в упорядоченном/разупорядоченном нестехиометрическом кобальтате Sr-Gd методом синхронного термического анализа. Журнал Сибирского федерального университета. Серия Химия. 2017. T. 10(3), C. 346-357. [Vereshchagin S., Dudnikov V., Solovyov L. Study of mobile oxygen in ordered/ disordered nonstoichiometric Sr-Gd-cobaltate by synchronous thermal analysis. Journal of Siberian Federal University. Chemistry 2017. Vol. 10(3), P. 346-357. (in Russ.)]

$$
-516-
$$


11. Vereshchagin S.N., Dudnikov V.A., Shishkina N.N., Solovyov L.A. Phase transformation behavior of $\mathrm{Sr} 0.8 \mathrm{Gd} 0.2 \mathrm{CoO} 3-\delta$ perovskite in the vicinity of order-disorder transition. Thermochimica Acta 2017. Vol. 655, P. 34-41.

12. James M., Cassidy D., Goossens D.J. and Withers R.L. The phase diagram and tetragonal superstructures of the rare earth cobaltate phases $\operatorname{Ln}_{1-x} \mathrm{Sr}_{x} \mathrm{CoO}_{3-\delta}\left(L n=\mathrm{La}^{3+}, \mathrm{Pr}^{3+}, \mathrm{Nd}^{3+}, \mathrm{Sm}^{3+}, \mathrm{Gd}^{3+}, \mathrm{Y}^{3+}\right.$, $\mathrm{Ho}^{3+}, \mathrm{Dy}^{3+}, \mathrm{Er}^{3+}, \mathrm{Tm}^{3+}$ and $\mathrm{Yb}^{3+}$ ). J. Solid State Chem. 2004. Vol. 177, P. 1886-1895.

13. Illers K.-H. Die ermittlung des schmelzpunktes von kristallinen polymeren mittels wärmeflusskalorimetrie (DSC). European Polymer Journal 1974. Vol. 10, P. 911-916.

14. Shiiba Hiromasa, Bishop C.L., Rushton M.J.D., Nakayama Masanobu, Nogami Masayuki, Kilner J.A. and Grimes R.W. Effect of A-site cation disorder on oxygen diffusion in perovskite-type $\mathrm{Ba}_{0.5} \mathrm{Sr}_{0.5} \mathrm{Co}_{1-\mathrm{x}} \mathrm{Fe}_{\mathrm{x}} \mathrm{O}_{2.5}$. Journal of Materials Chemistry A 2013. Vol. 1, P. 10345- 10352.

15. Vereshchagin S.N., Solovyov L.A., Rabchevskii E.V., Dudnikov V.A., Ovchinnikov S.G. and Anshits A.G. Methane oxidation over A-site ordered and disordered $\mathrm{S}_{\mathrm{r} 0.8} \mathrm{G}_{\mathrm{d} 0.2} \mathrm{Co}_{\mathrm{O} 3-\delta}$ perovskites. Chemical Communications 2014. Vol. 50, P. 6112-6115.

16. Kruidho F.H., Bouwmeester H.J.M., Doorn R.H.E. and Burggraaf A.J. Influence of orderdisorder transitions on oxygen permeability through selected nonstoichiometric perovskite-type oxides. Solid State Ionics 1993. Vol. 63-65, P. 816-822.

17. Isupova L.A., Alikina G.M., Tsybulya S.V., Boldyreva N.N., Kryukova G.N., Yakovleva I.S., Isupov V.P., Sadykov V.A. Real structure and catalytic activity of $\mathrm{La}_{1-\mathrm{x}} \mathrm{Sr}_{\mathrm{x}} \mathrm{CoO}_{3}$ perovskites. International Journal of Inorganic Materials 2001. Vol. 3, P. 559-562. 